\title{
The Investigation of the Obstacle Avoidance for Mobile Robot Based on the Multi Sensor Information Fusion technology
}

\author{
Qu Dongyue, Hu Yuanhang, and Zhang Yuting
}

\begin{abstract}
In order to accurately build a model about external environment of mobile robot, a robot multi-sensor system is designed. Ultrasonic sensor array adopt a distributed archi-tecture, infrared distance sensor use the design of class laser radar type, using multi-sensor information fusion technology, establish a $180{ }^{\circ}$ scrolling window in front of the robot, though the method of polar coordinates vector to obstacle detection and external environment model, using the "rolling window" obstacle avoidance algorithm, robots achieve autonomous obstacle avoidance and navigation in unknown environment. The experimental results confirmed that the robot system and the validity of the theory mentioned in this paper is valid and optimal.
\end{abstract}

Index Terms-Mobile robot, communication fusion, polar coordinate vector, rolling window.

\section{INTRODUCTION}

Sensor technology is widely applied in the field of intelligent robotics research [1] .There is easily interference by the environment in single sensor which causes large errors and the limited detection range [2]. Multi-sensor information fusion technology can achieve the goals of being complementary advantages of sensors and reduce redundant information; meanwhile it can improve the decision-making and respond ability of the system. So multi-sensor information fusion technology has broad application prospect [3].

At the present stage, multi-sensor information fusion technology mainly relies on the theoretical algorithm, such as Kalman filtering method, Bayesian method, least squares method[4], but less for real applications. Based on the practicality, an autonomous navigation robot is developed. To build an accurate model of the external environment, the robot multi-sensor system with ultrasonic sensors, infrared distance sensor, electronic comp-ass sensor and odometeris designed. Ultrasonic array has the distributed architecture, and the infrared distance sensor has the class laser radar type design, which realizes sensor multi-species, the number of information fusion. The experimental results demonstrate the effectiveness and practicality of the system designed [5].

Manuscript received December 28, 2012; revised February 2, 2013. Sources of project: the freedom to explore of central university research business (HEUCF100709).

Qu Dongyue and Hu Yuanhang are now with the Harbin Engineering University, China (e-mail: qudongyue@hrbeu.edu.cn, huyuanhanghgc@163.com).

Zhang Yuting is with the Institute of Advanced Technology of Heilongjiang Academy of Science, China.

\section{AutonOMOUS NAVIGATION RoBOt SySTEM DESIGN}

In this study, to realize the autonomous robot control and navigation, the main control center of the designed autonomous navigation robot is a personal computer IPC, which uses the $\mathrm{C}++$ language to write the software program by considering the stability, dynamics and optimization. The robot has four parts:

1) Energy layer. The layer is powered by a $12 \mathrm{~V}, 2400 \mathrm{~mA}$ lithium battery-powered.

2) Driver layer. Mobile robot by Sabertooth dual 10A DC motor drive provides up to $10 \mathrm{~A}$ of continuous driving, meanwhile using four $12 \mathrm{~V}$ Coreless planetary gear motor.

3) Sensor layer. The sensor layer has six ultrasonic sensors, an infrared distance sensor, an electronic compass sensor and an odometer.

4) Master layer. The master layer is composed of 1 personal computer PC, which can complete the acquisition of sensor information, multi-sensor information fusion, the planning algorithm processing and other functions.

The autonomous navigation robot in have the following characteristics: The action of the robot is flexible and reliable and the movement lines are smooth and continuous:The overall structure of the robot is taken by modular design, which is easy to control and achieve.

\section{Multi-SENSOR INFORMATION FUSION SYSTEM}

Multi-sensor information fusion technology is an effective information-processing technology, which has a multi-sensor type, number of collaboration achieving complementary advantages of the sensor, high system reliability and strong anti-interference ability.

The mobile robot selects ultrasonic sensors and infrared range sensors as distance detector. The ultrasonic sensor has large measuring range, but it has a "cross-talk" feature and is easy to produce "phantom" phenomenon, which could easily cause large accidental errors [6]. Infrared distance sensor can compensate the ultrasonic sensors for the blind spots, but it has the smaller measuring range. So the ultrasonic sensors and infrared range sensors exchange information to realizing complementary advantages and a more accurate external environment model [7].

\section{A. Ultrasonic Sensors Distributed System}

To the goal of autonomous obstacle avoidance and navigation, the mobile robot needs comprehensively survey of the external environment. External environment of robot is divided into two parts: $180^{\circ}$ front area and $180^{\circ}$ back area. In the process of robot autonomous navigation, the front 
area of $180^{\circ}$ is inevitable detection area. In this paper, six ultrasonic sensors are used to detect the front of the robot $180^{\circ}$ area. If concave trap and local extremum are encountered, robot will spin around $180^{\circ}$ and change its front and rear, then the new path will be planned again.

Ultrasonic sensors are detected in a distributed installation method and every sensor has $60^{\circ}$ cone angle, Right horizontal line as a reference line, the face perpendicular to the center line of ultrasonic sensors B, C, D, $\mathrm{E}, \mathrm{F}, \mathrm{G}$ and reference line sequentially into a $30^{\circ}, 60^{\circ}, 90^{\circ}$, $90^{\circ}, 120^{\circ}, 150^{\circ}$ installation, the sensor arrangement shown in Fig. 1:

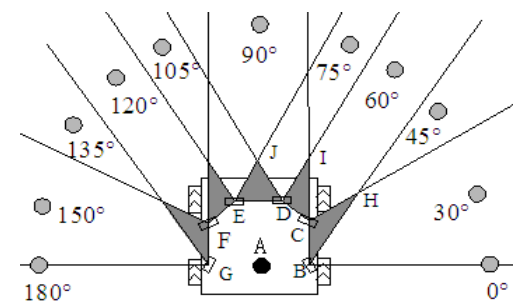

Fig. 1. Arrangement of ultrasonic sensors and judgment about azimuth of obstacles.

In Fig. 1, each sensor emits two rays representation of detected region boundary lines, short black dots indicate the obstacle. The angles in the chart are the azimuth angle of the obstacle that identified by two adjacent sensors. The detection range of optional ultrasonic sensor is $4 \mathrm{~cm}-160 \mathrm{~cm}$ The black triangle area is blind spots of detection about two adjacent ultrasonic sensors, and the area is detected and added by Infrared ranging sensors. Based on the ultrasonic sensor array obstacle azimuth, the practice is shown below:

The distance values measured by the six ultrasonic sensors are represented by $L_{i}(1 \leq i \leq 6)$, which is in the range of $4 \mathrm{~cm}-160 \mathrm{~cm}$. It shows a maximum 160 when the ultrasonic sensor cannot detect obstacles, the actual distance between the obstacles to the robot is represented by $L_{d}$, the angle of the obstacle is represented by $\alpha_{d}$. The angles of 6 ultrasonic sensors arranged on the robot are known, using the number of the two adjacent ultrasonic sensors to show the obstacle angle information, just as the picture 1 shown. Establish the basic rules libraries as follows:

$i$ : If $P$ then $Q$

$i$ indicates $i$-th rule, $P$ represents the conditions of the rule, $Q$ represents the results of the rules.

1) If $4 \leq L_{1} \leq 160$ and $L_{2}=160$ then $L_{d}=L_{1}$ and $\alpha_{d}=30^{\circ}$;

2) If $4 \leq L_{1}<160$ and $4 \leq L_{2}<160$ then $L_{d}=\min \left(L_{1}, L_{2}\right)$ and $\alpha_{d}=45^{\circ}$;

$3)$ If $4 \leq L_{2}<160$ and $L_{3}=160$ then $L_{d}=L_{2}$ and $\alpha_{d}=60^{\circ}$;

4) If $4 \leq L_{2}<160$ and $4 \leq L_{3}<160$ then $L_{d}=\min \left(L_{2}, L_{3}\right)$ and $\alpha_{d}=75^{\circ}$;

5) If $4 \leq L_{3}<160$ and $4 \leq L_{4}<160$ then $L_{d}=\min \left(L_{3}, L_{4}\right)$ and $\alpha_{d}=90^{\circ}$;

6) If $4 \leq L_{4}<160$ and $4 \leq L_{5}<160$ then $L_{d}=\min \left(L_{4}, L_{5}\right)$ and $\alpha_{d}=105^{\circ}$;

7) If $4 \leq L_{5}<160$ and $L_{4}=160$ then $L_{d}=L_{5}$ and $\alpha_{d}=120^{\circ}$;
8) If $4 \leq L_{5}<160$ and $4 \leq L_{6}<160$ then $L_{d}=\min \left(L_{5}, L_{6}\right)$ and $\alpha_{d}=135^{\circ}$;

9) If $4 \leq L_{6}<160$ and $L_{5}=160$ then $L_{d}=L_{6}$ and $\alpha_{d}=150^{\circ}$.

The subject selected six ultrasonic sensors to arrange which can approximately get the information of nine azimuths. It's sure that you can set more ultrasonic sensors. With the increasing number of sensors, the accuracy of judging the obstacle azimuth will increase.

\section{B. Establish Obstacle Model with Polar Coordinate Vectors}

Establishing obstacle model with polar coordinates vector method is an improvement of grid method[8]. The principle is to store the external characteristics of the obstacle in the form of direction vector, but only to do this. It overcomes the shortcomings of the traditional grid method, such as large reserves and long cycle modeling, etc.

The method to create a polar coordinate vector is as follows: firstly, divide the $180^{\circ}$ rolling window into $\mathrm{n}$, and then establish the vector window made up of $180^{\circ} /(n+1)$ vector line to establish obstacle feature model by the vector lines. Of course, the greater the value of $n$, the more intensive the vector is and the more accurate the model is, but the amount of data that need to be handled also increases. The merit of establishing is using a line instead of a bunch of grid ${ }^{[8]}$. And the selection of $\mathrm{n}$ should follow the principle that each grid is mapped at least one vector line. $\delta$ should satisfy the following equation:

$$
\begin{gathered}
\delta=180^{\circ} /(n+1) \\
2 R^{2}-2 R^{2} \times \cos \delta \leq \sqrt{2} S^{2}
\end{gathered}
$$

$\delta$ is the angle two adjacent vector lines. $R$ is the threshold of scrolling window .S is the length of the grid side.

The grid size in this subject is $16 \times 16 \mathrm{~cm}$, the threshold of scrolling window is $60 \mathrm{~cm}$.Having calculated, $\delta$ can take as $18^{\circ}$, so it can create 11 direction vectors. Now we assume the length of $i$-th vector as $d_{i}$, if $d_{i}<R$, the vector will be an effective vector. The start angle of rolling window is $0^{\circ}$, step angle $\theta$ is $18^{\circ}$, the direction of the vector angles are determined as follows: $\alpha_{d 1}=0^{\circ}, \alpha_{d 2}=18^{\circ}, \alpha_{d 3}=36^{\circ} \cdots$ $\alpha_{d 11}=180^{\circ}$, and ultimately we create a direction vector $\vec{L}\left(\alpha_{d i}, d_{i}\right)$ collection as the obstacles contour curves, the range of $i$ is $1 \leq i \leq 11$. Of course, the number of vector line $\mathrm{n}$, environmental modeling more accurately, the corresponding information processing capacity is greater. Polar coordinates vector method to establish the principle of the obstacle model shown in Fig. 2:

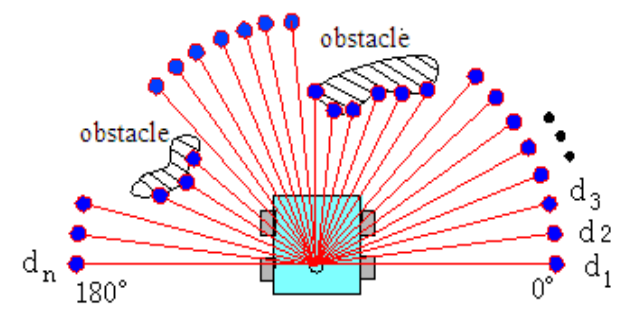

Fig. 2. The establishment of the polar coordinates Vector 
The advantage of establishing obstacle model with polar coordinates vector method is that accurate model, small reserves, fast response of robot.

\section{Design of Infrared Ranging Sensor a "Class Laser Radar"}

Establishing obstacle model with polar coordinates vector method requires infrared ranging sensor system to create a $180^{\circ}$ rolling window in front of the robot and simultaneously detect the distance and azimuth angle of the obstacle. To the above requirement, a "class laser radar" system based on infrared ranging sensor is designed. Specifically as follows: install a infrared ranging sensor to steering gear head, detect by the infrared ranging sensor, get feedback angle by steering gear head. Eventually real-time detection of parameters of obstacle and azimuth are achieved. Infrared distance sensor system installed position of point A in Fig. 2.

The "class laser radar" system consists of three parts: 1) pan-tilt 2) infrared ranging sensor 3 ) the main control center. The infrared ranging sensor is mounted to the $180^{\circ}$ rotation of the steering gear head, and the steering gear rotation is accurately controlled by USBSS32 control panel.USBSS32 goes on data communications via USB interface with PC, which can implement the setting of the scan initialization, stop scanning, etc. The main control center uses a PC . Each time it deals with the distance information measured by the rotation angle and the infrared ranging sensor of the steering gear PC as a simultaneous acquisition and storage, after analysis and processing of data, the external environment information is defined.

There are ten pulse angle of the steering gear in this study, which can complete exploration mission well. With the increasing number of servos pulse width, the number of rolling window polar coordinates vector line $n$ also increases, and the angular resolution is higher. But the probe cycle will be longer. This should be determined according to the actual situation.

According to the above methods, establishing a "class laser radar" system with the application of infrared ranging sensor and building the $180^{\circ}$ rolling window in front of the robot can accurately establish the obstacle environment model in polar coordinates vector method ${ }^{[10]}$.

\section{Divide of Multi-Sensor Information Fusion Level}

The strategy of mobile robot motion is divided into four levels: the layer that tend to target; reduction layer; the layer of accurate response; the layer of emergency response. The function of each level is as follows:

The layer that tend to target: When Sensor system does not detect obstacles, the robot executes "Strategies tend to target".

Reduction layer: When the ultrasonic probe area detect obstacle first, the robot performs "reduction strategy" to prepare for the implementation of the follow-up obstacle avoidance strategy.

The layer of accurate response: When obstacle is detected by the Common detection area, the robot performs accurate obstacle avoidance strategy. The layer is the key one to the robot obstacle avoidance system.

The layer of Emergency response: When in an emergency, such as the robot encounters concave trap, local extreme, the robot can not avoid obstacles in precise planning layer, then the robot perform emergency obstacle avoidance strategy.

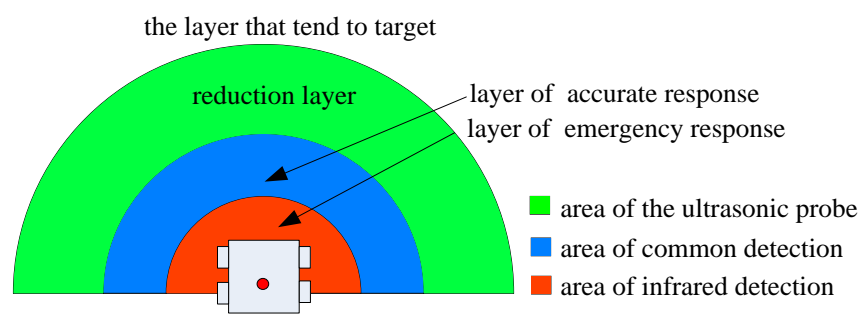

Fig. 3. Hierarchical division of multi-sensor information fusion

In the four levels, the layer of accurate response is the core part of the levels. So the following is the studying about the scoping of the accurate response layers .

The layout of Sensor is shown in Figure 1. A is the infrared distance sensor; every one of B, C, D, E, F and G is an ultrasonic sensor, and they are putted in symmetrical arrangement. H, I, J are the vertices of the detecting blind spot of the two adjacent sensor. Calculate AH, AL, AJ and choose the maximum value as the minimum radius for blind spot detection complemented by infrared ranging sensor and the defined line which is the common detection area of ultrasonic sensor and infrared ranging sensor.

Known: The length of robot is $36 \mathrm{~cm}$, and width is $30 \mathrm{~cm}$. $\mathrm{A}, \mathrm{B}, \mathrm{G}$ are collinear, and they has a $8 \mathrm{~cm}$ distance from the bottom line. $\mathrm{D}$ and $\mathrm{E}$ are collinear and a $4 \mathrm{~cm}$ distance from the top line. $A B=15 \mathrm{~cm}, \quad B C=16 \mathrm{~cm}, \quad D E=14 \mathrm{~cm}$. $\angle C B H=30^{\circ}, \angle J D E=60^{\circ}$ Through mathematical calculations: $\mathrm{AH}=37.35 \mathrm{~cm}, \mathrm{AI}=40.71 \mathrm{~cm}, \mathrm{AJ}=36.12 \mathrm{~cm}$. So select the maximum value of $40.71 \mathrm{~cm}$ for starting boundary of the common detection area.

In this paper, the detection range of the Infrared Ranging sensor is from $10 \mathrm{~cm}$ to $80 \mathrm{~cm}$. The detection range of the ultrasonic sensor is from $4 \mathrm{~cm}$ to $160 \mathrm{~cm}$. Infrared distance sensor in installation position of $\mathrm{A}$ as the reference. With the above analysis, we can determine the range of the infrared detection that from $10 \mathrm{~cm}$ to $40.71 \mathrm{~cm}$. The structural dimensions of the robot just to make up Infrared ranging sensors detect blind spots. A large number of experiments confirmed that: $80 \mathrm{~cm}$ is most appropriate to the division of reduction layer and the layer of accurate response should. So the area of Common detection is $40.17 \mathrm{~cm} \sim 80 \mathrm{~cm}$, the area of the ultrasonic probe is $80 \mathrm{~cm} \sim 160 \mathrm{~cm}$.

The point of infrared ranging sensors located as a reference. Every level is as follows considering the robot dimensional bodywork:

The layer of Emergency response: $<40.71 \mathrm{~cm}$;

The layer of Accurate response: $40.71 \mathrm{~cm} \sim 80 \mathrm{~cm}$;

Reduction layer: $80 \mathrm{~cm} \sim 160 \mathrm{~cm}$;

The layer that Tend to target: $>160 \mathrm{~cm}$.

Experiments show that the design of mobile robot's various levels makes the robot more stable performance, and the detection result will be more accurate

\section{OBSTACLE DETECTION AND EXTERNAL ENVIRONMENT MODELING}

Mobile robot establishes rolling window, then obstacles polar diagram is set up by the contrast obstacle detection distance and the valve value $\mathrm{R}$ of rolling window. Based on 
the analysis and processing of polar coordinates vector graph data, the obstacles of the characteristic parameters are determined including obstacles starting angle, end angle and the minimum distance of the obstacle to robot reference point A. All the information above can determine the basic information of an obstacle.

In the detection process of robot obstacles, firstly the concept of "bounding box" is introduced. That is: In mobile robot motion process, when found that obstacles implement obstacles avoidance strategy, the obstacle model should be expanded treatment at first to prevent collision between the robot and obstacles edge. The expanded virtual obstacle model is called the bounding box, and the distance between the edge and the original obstacles is $\Delta d$.The bigger $\Delta d$ is, the higher obstacle avoidance safety coefficient is.

When $d_{i}<R$, rolling window will be considered that there are obstacles exist inside in the process of detecting obstacle. The discrimination of obstacles types in robot system can be divided into five kinds as follows:

1) When $d_{i} \leq R, d_{i+1} \leq R$ and $\left|d_{i+1}-d_{i}\right|<L$, the system determines the same obstacle and the detection continues.

2) When $d_{i} \leq R, d_{i+1} \leq R$ and $\left|d_{i+1}-d_{i}\right| \geq L$, the system determines two obstacles overlapping.

3) When $d_{i} \leq R$ and $d_{i+1}>R$, the system determines that a obstacle detection has ended.

4) When $d_{i}>R$ and $d_{i+1} \leq R$, the system determines that a new obstacle detection starts.

5) When $d_{i}>R$ and $d_{i+1}>R$, the system determines that there are no obstacle around the robot.

Among them, $d_{i}$ is the length of article $\mathrm{i}$ vector line in rolling window, $R$ is a threshold of rolling window. $L$ is the safety through the spacing of robot, $L=D_{R}+2 \Delta d, D$ is the width of the robot body , $\Delta d$ is the puffed distance of obstacle.

Mobile robot using polar coordinates vector method to establish models of obstacle process as shown in figure 5:

Autonomous navigation robot through the rolling window to establish external environment model, and can achieve obstacle detection and obstacle overlap the complicated situation of judgment, then we can well establish external environment model, for the robot autonomous obstacleavoiding and navigation ready.

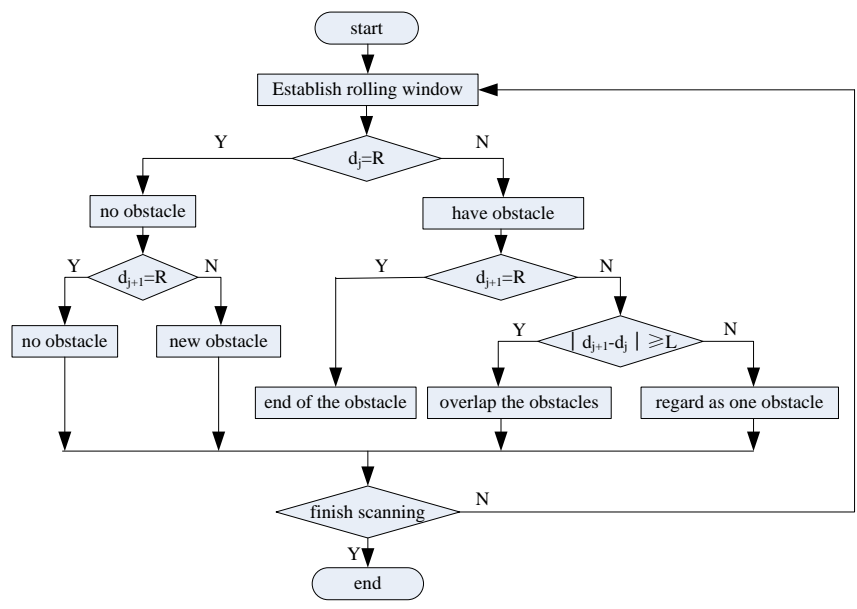

Fig. 4. Detecting obstacles and modeling flow chart

\section{EXPERIMENTS ABOUT OBSTACLE AVOIDANCE OF THE AUTONOMOUS NAVIGATION ROBOT}

This paper designed autonomous mobile robot, established the ultrasonic ranging sensor system, infrared distance sensor "laser radar" system, using multi sensor information fusion technology, combined with the polar coordinate vector method to establish the environmental model, robot obstacle avoidance algorithm applied to scroll the window of obstacle avoidance and navigation. The following experiment is used MATLAB simulation and robot platform to verify the validity of the proposed theory.

In the MATLAB simulation environment, the model shown in Figure 5: in the figure, the red represents the mobile robot, in front of the red establish a $180^{\circ}$ scrolling window, the corridor is the both sides of the black part, the black middle part is the obstacle model

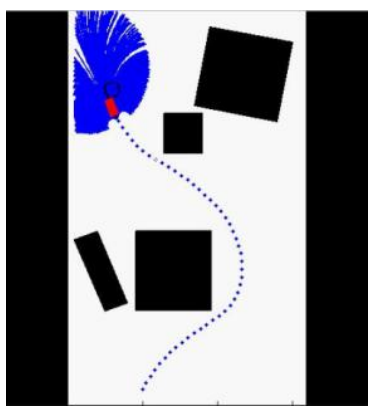

Fig. 5. Simulation about autonomous navigation

From the figure can be seen, the mobile robot has completed the path selection from the start point to the target point, and successfully avoided the obstacle encountered by the advance, and the robot path selected is feasible and optimality. Confirming the effectiveness of mentioned algorithm[9]

In the research, we use the platform of autonomous mobile robot to do obstacle avoidance experiment. In the experiment, we use three cartons and one finishing tank as the obstacles. the export on the left of finishing box is the target position of the robot. Figure 6 is the process of autonomous mobile robot autonomous navigation.
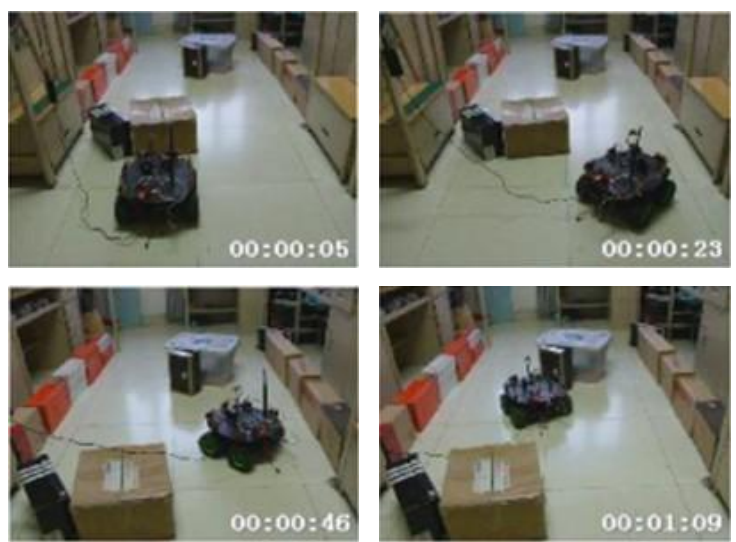

Fig. 6. Obstacle avoidance experiments with many obstacles

Robot during exercise, ultrasound detection zone first detects obstacles, robot in the deceleration chronic mode. The robot move on, enter the exact planning layer, starting a real-time obstacle avoidance strategy, the robot avoid obstacles encountered, eventually reaching the target point. As can be seen from the figure, the robot independently choose the path of feasibility, optimization, and confirmed 
the excellent characteristics of the proposed method.

\section{CONCLUSION}

An autonomous mobile robot, composed of energy layer, driver, sensor layer and control layer, was designed in this paper. The ultrasonic ranging sensor system and infrared distance sensor "laser radar" system were established in the robot. The technology of multi-sensor information fusion was applied. $180^{\circ}$ rolling window was built in front of the robot, which could be divided into the trend of the target layer, slow down layer, precise planning layer and emergency planning layer. Combined with polar coordinates vector method for obstacle detection and the external environment model, the robot can precisely detect the environment. The algorithm of the "rolling window" obstacle avoidance was employed to realize the autonomous obstacle avoidance and navigation of the robot in an unknown environment. The effectiveness and the optimization of the robot designed system and the presented theory were validated by the results of the experiments in this project.

\section{REFERENCES}

[1] L. Ren, "Obstacle Perception and Obstacle-avoiding Strategy Pesearch of Mobile Robot Based On Laser Range Finder," Harbin: Harbin Institute of Technology, 2007.

[2] H. A. Hagras, "A Hierarchical Type22 Fuzzy Logic Cont rol Architecture for Autonomous Mobile Robot," IEEE Trans. actions on Fuzzy Systems, vol. 12, no. 4, pp. 524-539, August 2004.

[3] Z. Ji, "Mobile Robot Target Detection and Target Recognition Fusion Technology Research,” Jinan: Shandong University, 2011.

[4] J. Bai, L. Chen, H. Jin, R. Chen, and H. Mao, "A new path planning for robot in dynamic and unknown environments," Transducer and Microsystem Technologies, vol. 30, no. 10, pp. 33-36, 2011.

[5] S. Kim and Y. Kim, "Robot Localization Using Ultrasonic Sensors," in Proc. IEEE/SRJ International Conference on Intelligent Robots and Systems, Sendai, Japan, 2004, vol. 4, pp. 3762-3766.
[6] H. Boubertakh, M. Tadjine, and P. Y. Glorennec, "A new mobile robot navigation method using fuzzy logic and a modified Q-learning algorithm," Journal of Intelligent Fuzzy Systems, vol. 21, pp. 113-119, 2010.

[7] F. Niu, "Research on the Multi-mode Control System of Intelligent Wheelchair," Nanchang: Nanchang University, 2009.

[8] B. Sun, D. Han, and Q. Wei, "Application of Rolling Window Algorithm to the Robot Path Planning," Computer Simulation, vol. 23, no. 06 , pp. 159-162, 2006.

[9] J. L. Blanco and J. Gonzale, and J. A. Fernandez Madriga, "Extending obstacle avoidance methods through multiple parameter space transformations," Autonomous Robots, vol. 24, no. 01, pp. 29-48, 2008 .

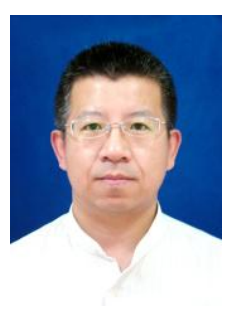

Qu Dongyue was born in 1973, who graduated from the Moscow State the Process University STAKIN “ in 2008. He has obtained his Ph.D degree. He is now working at Harbin Engineering University. The main research areas are mobile robot control technology

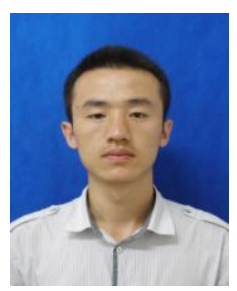

Hu Yuanhang was born in 1986. He is now studying at the Harbin Engineering University, his current research areas aremobile robot sensing and control technology.

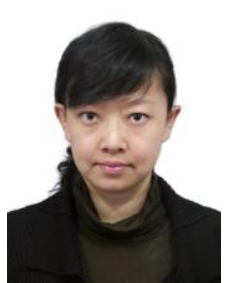

Zhang Yuting was born in 1976. She graduated from the Moscow the State Process University "STAKIN in 2006. Her current research areas are modern industrial technology. 